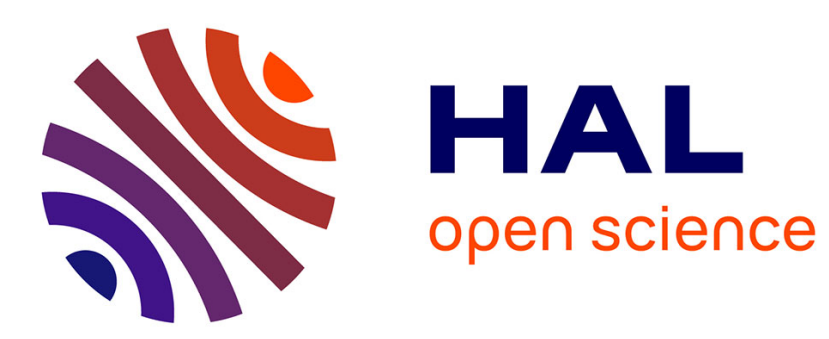

\title{
Retraite par répartition ou par capitalisation: une analyse de long terme
}

\author{
Gabrielle Demange, Laroque Guy
}

\section{To cite this version:}

Gabrielle Demange, Laroque Guy. Retraite par répartition ou par capitalisation: une analyse de long terme. Revue Economique, 2000, 51 (4), pp.813-829. halshs-00585272

\section{HAL Id: halshs-00585272 \\ https://shs.hal.science/halshs-00585272}

Submitted on 12 Apr 2011

HAL is a multi-disciplinary open access archive for the deposit and dissemination of scientific research documents, whether they are published or not. The documents may come from teaching and research institutions in France or abroad, or from public or private research centers.
L'archive ouverte pluridisciplinaire HAL, est destinée au dépôt et à la diffusion de documents scientifiques de niveau recherche, publiés ou non, émanant des établissements d'enseignement et de recherche français ou étrangers, des laboratoires publics ou privés. 


\title{
Retraite par répartition ou par capitalisation. Une analyse de long terme
}

In: Revue économique. Volume 51, n4, 2000. pp. 813-829.

\begin{abstract}
Résumé
Un large débat s'est ouvert dans les pays occidentaux pour modifier les systèmes de retraite basés sur la répartition. II est souvent malaisé de déterminer les mécanismes à l'œuvre dans les études récentes, à base de simulations, qui cherchent à quantifier les enjeux. Nous espérons ici clarifier les effets à long terme d'une modification des régimes de retraite. Dans le cadre d'une économie à générations imbriquées, nous évaluons l'impact sur les équilibres stationnaires des variations du taux de cotisation à un système de répartition avec différentes politiques de financement des dépenses publiques.
\end{abstract}

\section{Abstract}

Pay as you go or fully funded social security: a long run analysis?

There is a lot of pressure in the developed countries to modify the pay-as-you-go social security systems. It is often unclear to separate what is important from what is accessory in recent simulation studies, which depend on precise assumptions regarding the demographic trends or taxes. We try to clarify the debate using a simple theoretical overlapping generations model. We analyze the comparative statics of the long run stationary equilibria with respect to the contribution rates to the pay as you go system, under various financing arrangements, through public debt, a wage tax or a tax on interest income.

Citer ce document / Cite this document :

Demange Gabrielle, Laroque Guy. Retraite par répartition ou par capitalisation. Une analyse de long terme. In: Revue économique. Volume 51, $\mathrm{n}^{\circ} 4,2000$. pp. 813-829.

http://www.persee.fr/web/revues/home/prescript/article/reco_0035-2764_2000_num_51_4_410556 


\title{
Retraite par répartition ou par capitalisation
}

\author{
Une analyse de long terme
}

\author{
Gabrielle Demange* \\ Guy Laroque
}

\begin{abstract}
Un large débat s'est ouvert dans les pays occidentaux pour modifier les systèmes de retraite basés sur la répartition. II est souvent malaisé de déterminer les mécanismes à l'ceuvre dans les études récentes, à base de simulations, qui cherchent à quantifier les enjeux. Nous espérons ici clarifier les effets à long terme d'une modification des régimes de retraite. Dans le cadre d'une économie à générations imbriquées, nous évaluons l'impact sur les équilibres stationnaires des variations du taux de cotisation à un système de répartition avec différentes politiques de financement des dépenses publiques.
\end{abstract}

\section{PAY AS YOU GO OR FULLY FUNDED SOCIAL SECURITY: A LONG RUN ANALYSIS?}

There is a lot of pressure in the developed countries to modify the pay-asyou-go social security systems. It is often unclear to separate what is important from what is accessory in recent simulation studies, which depend on precise assumptions regarding the demographic trends or taxes. We try to clarify the debate using a simple theoretical overlapping generations model. We analyze the comparative statics of the long run stationary equilibria with respect to the contribution rates to the pay as you go system, under various financing arrangements, through public debt, a wage tax or a tax on interest income.

Classification JEL : E2, E6, H3

Un large débat s'est ouvert dans les pays occidentaux pour modifier les systèmes de retraite basés sur la répartition. Une des préoccupations principales porte sur l'avenir des systèmes actuels gravement touchés par l'évolution démographique : baisse du taux de fécondité, allongement de la durée de vie, retard de l'entrée dans la vie active. Toutes les projections montrent qu'il est impossible de maintenir les taux de remplacement sans augmenter sensiblement les taux de cotisation et/ou augmenter la durée de vie active.

Indépendamment de cette évolution, d'autres critiques sont avancées contre la répartition, tout particulièrement aux États-Unis. Elle introduirait, de par son

* DELTA, ENS 48 boulevard Jourdan, 75014 Paris, France.

** INSEE, Direction des Études et Synthèses économiques 15, boulevard GabrielPéri, BP 100 - G 001, 92244 Malakoff Cedex.

Cet article a bénéficié des commentaires de deux lecteurs anonymes. 
financement parafiscal, une distorsion sur l'offre de travail. Elle réduirait l'épargne des ménages et conduirait à un sous-investissement. Ce dernier argument s'appuie parfois sur l'observation des rendements élevés du capital, qui sont supérieurs à ce qui ressortirait de la règle d'or des modèles de croissance (Abel et al. [1989]). Pour quantifier les enjeux, plusieurs études, à base de simulations, ont été menées dans divers pays (par exemple Bailen et Gil [1996] pour l'Espagne, Feldstein et Samwick [1996,1997], Huang, Imrohoroglu et Sargent [1996], Kotlikoff, Smetters et Walliser [1996] pour les États-Unis). Elles tentent d'évaluer les gains à espérer d'un système de retraite par capitalisation et de mettre en évidence des scénarios où la transition de la répartition à la capitalisation serait suffisamment peu douloureuse pour être politiquement acceptable. Même si ces études concluent souvent en faveur du passage à la capitalisation, elles diffèrent sensiblement aussi bien par l'évaluation des gains de long terme que par les moyens à mettre en cuvre durant la transition.

Il est souvent malaisé de distinguer dans les modèles de simulation, au travers des diverses modalités de représentation des évolutions démographiques et d'évolution du système fiscal, les mécanismes à l'œuvre et les hypothèses qui sont essentielles. Nous essayons ici d'apporter quelques éléments de clarification en nous appuyant sur un modèle théorique simple. Il est basé sur une économie à la Samuelson avec des générations imbriquées, ce qui permet l'étude des transferts intergénérationnels. Le modèle doit être enrichi de façon à traiter des retraites par répartition ou par capitalisation, et des modalités de leur financement. Aussi introduisons-nous la production, comme dans Diamond [1965], et les dépenses publiques.

Nous nous limitons à l'étude des équilibres stationnaires (ou de croissance régulière), associés à des taux d'imposition, un niveau de dépenses, de dette publique par tête constant. Un changement de régime conduit l'économie vers un nouvel état d'équilibre de long terme. L'analyse théorique permet de classer les divers équilibres de l'économie (il y en a plusieurs possibles) et indique comment ils varient avec le taux de cotisation du système de répartition. Elle permet de mieux comprendre les problèmes d'éviction : la capitalisation n'implique pas nécessairement un stock de capital d'équilibre plus élevé que la répartition. Au vu de nos résultats théoriques, nous examinons les simulations de Feldstein et Samwick [1996, 1997]. Ces auteurs cherchent à évaluer les gains de long terme à attendre du passage à la capitalisation aux États-Unis.

L'article est organisé en trois parties. Les deux premières parties présentent le modèle théorique et formalisent les éléments essentiels du débat. La troisième cherche à chiffrer les principaux effets mis en évidence sur le modèle théorique et fait le lien avec les études de Feldstein et Samwick.

\section{LE MODĖLE THÉORIQUE}

Le modèle théorique très schématique sur lequel on va raisonner a une structure voisine de celui de Diamond [1965]. Les agents, producteurs et consommateurs, ont des comportements de concurrence parfaite. Il existe un régime de retraite par répartition obligatoire et des prélèvements fiscaux qui financent les dépenses de l'État. Pour éviter d'alourdir les notations, les agents vivent deux 
périodes, offrent leur travail de façon inélastique lorsqu'ils sont jeunes et il n'y a pas de progrès technique ${ }^{1}$. Tous les résultats se généralisent au cas où la durée de vie du consommateur représentatif est finie ${ }^{2}$. La population croît au taux constant $n, n \geq 0$, et nous examinerons des trajectoires régulières le long desquelles les grandeurs par tête sont constantes.

\section{La production et les prix}

La production s'effectue à rendements constants à partir du stock de capital hérité de la période précédente et du travail des jeunes. Aussi peut-on raisonner sur les quantités par tête : si $k$ est le stock de capital par tête de jeune en début de période, $f(k)$ est la production brute par tête ${ }^{3}$. Comme il est usuel nous supposons :

HYPOTHEेSE 1: La production brute par tête $f$ est concave. De plus $\lim _{k \rightarrow 0} f^{\prime}(k)=\infty, \lim _{k \rightarrow \infty} f^{\prime}(k)=0$.

Lors de la production, le capital s'use au taux $\delta, \delta>0$, par période : pour un input de $k$ en entrée du système productif, on récupère $f(k)+(1-\delta) k$ en fin de période.

On se place dans un cadre de concurrence parfaite où le salaire et le taux d'intérêt sont donnés par les productivités marginales :

$$
w_{t}=f\left(k_{t}\right)-k_{t} f^{\prime}\left(k_{t}\right), \quad r_{t}=f^{\prime}\left(k_{t}\right)-\delta
$$

L'hypothèse de rendements constants entraîne :

$$
f\left(k_{t}\right)=w_{z}+\left(r_{t}+\delta\right) k_{t}
$$

\section{Le consommateur}

Le salaire est taxé au taux $\tau_{l}$ et les revenus de l'épargne au taux $\tau_{k}$. Pour garder une présentation simple, on supposera $\tau_{l}$ et $\tau_{k}$ constants et donnés. L'agent peut placer son épargne sous deux formes : capital physique et bons du Trésor. En l'absence d'aléas et de contraintes d'endettement, ces deux placements ont même rendement avant impôt $r$. La retraite est versée proportionnellement aux cotisations prélevées sur le travail. On la paramètre avec le taux de remplacement $\theta$ : en régime stationnaire, le taux de cotisation est $\theta /(1+n)$. En

1. Un progrès technique à taux constant, si les fonctions d'utilité sont homogènes, conduit à un modèle très semblable, une fois que l'on norme les quantités par unité efficace de la période de référence. Il suffit alors, en première approximation, d'ajouter le taux de croissance du progrès technique au taux de croissance de la population dans les formules qui caractérisent l'équilibre.

2. Lorsque la généralisation est immédiate, nous présentons l'argument avec le modèle à deux périodes. Sinon, comme c'est le cas de la proposition 2 ci-après, nous donnons une démonstration générale.

3. Avec des notations évidentes $k_{t}=\mathrm{K}_{t-1} / \mathrm{L}_{t}$ et $f\left(k_{t}\right)=\mathrm{F}\left(\mathrm{K}_{t-1}, \mathrm{~L}_{t}\right) / \mathrm{L}_{t}$ où $\mathrm{F}$ est la fonction de production globale. 
notant $c_{t}^{j}$ et $c_{t+1}^{\nu}$ la consommation aux deux périodes de la vie, le programme du consommateur type s'écrit :

$$
\left\{\begin{array}{l}
\max u\left(c_{t}^{j}, c_{t+1}^{v}\right) \\
c_{t}^{j}+s_{t}+b_{t}=\left(1-\tau_{l}\right)\left[w_{t}-\frac{\theta w_{t}}{1+n}\right] \\
c_{t+1}^{v}=s_{t}+b_{t}+\theta\left(1-\tau_{l}\right) w_{t+1}+r_{t+1}\left(1-\tau_{k}\right)\left(s_{t}+b_{t}\right)
\end{array}\right.
$$

où $s_{t}$ et $b_{t}$ sont les placements respectivement en capital physique et en bons du Trésor. S'il n'y a pas de restriction à l'endettement, les deux contraintes de budget se résument en une seule contrainte de budget intertemporelle :

$$
c_{t}^{j}+\frac{c_{t+1}^{v}}{1+r_{t+1}\left(1-\tau_{k}\right)}=W_{t}
$$

avec

$$
W_{t}=\left[w_{t}+\theta\left(-\frac{w_{t}}{1+n}+\frac{w_{t+1}}{1+r_{t+1}\left(1-\tau_{k}\right)}\right)\right]\left(1-\tau_{t}\right)
$$

\section{L'État}

En notant $g_{t}$ la dépense publique par tête d'agent jeune, la contrainte de budget du gouvernement s'écrit, toujours par tête d'agent jeune :

$$
g_{t}=\tau_{l} w_{t}+\tau_{k} r_{t} \frac{s_{t}-1}{1+n}-\frac{b_{t}-1}{1+n}\left(1+\left(1-\tau_{k}\right) r_{t}\right)+b_{t}
$$

Une fois fixé le paramètre $\theta$ qui décrit le niveau des retraites, les taux d'imposition $\tau_{l}, \tau_{k}$, il reste à chaque date un degré de liberté pour déterminer la politique du gouvernement, c'est-à-dire pour choisir $\left(g_{t}, b_{t}\right)$ qui vérifie la contrainte de budget. On peut considérer plusieurs types de comportements. Par exemple :

- l'État choisit un niveau de dépenses publiques par tête $g_{t}$ et ajuste en conséquence le niveau de sa dette $b_{t}$;

- il fixe un déficit primaire par tête $d_{t}=\tau_{l} w_{t}+\tau_{k} r s_{t-1}-g_{t}$, ajuste les dépenses en conséquences et fixe la dette publique de façon à satisfaire sa contrainte budgétaire.

Dans la suite, nous nous limiterons au premier cas en supposant que le niveau des dépenses publiques, supposé constant, n'est pas affecté par une modification du système de retraite. Une variation du taux de remplacement se traduira alors en général par une variation du déficit primaire. Ainsi, non seulement l'investissement pourra être modifié mais aussi le montant de la dette publique.

\section{L'équilibre emplois-ressources}

La contrainte emplois-ressources, écrite avec les quantités par tête d'agent jeune, s'écrit à la date $t$ :

$$
g_{t}+c_{t}^{j}+\frac{c_{t}^{\nu}}{1+n}+s_{t}=f\left(k_{t-1}\right)+(1-\delta) k_{t-1}
$$




\section{Équilibres stationnaires}

Nous nous intéresserons aux situations stationnaires. Le niveau de dépenses publiques, les taux d'imposition seront supposés constants ainsi que le taux de remplacement. Notre objectif est d'étudier les équilibres associés à ces valeurs $g, \tau_{l}, \tau_{k}, \theta$, et principalement de regarder comment ils varient avec le taux de cotisation ${ }^{1}$.

Le long d'une trajectoire stationnaire, les prix et les quantités par tête sont constantes. La contrainte de budget du gouvernement s'écrit :

$$
g=\tau_{l} w+\tau_{k} r \frac{s}{1+n}+b \frac{\left(n-r\left(1-\tau_{k}\right)\right)}{1+n}
$$

Il est commode de définir les fonctions $\mathrm{C}^{j}(r, \mathrm{~W})$ et $\mathrm{C}^{\nu}(r, \mathrm{~W})$, consommations du jeune et du vieux, solutions de :

$$
\left\{\begin{array}{l}
\max u\left(c^{j}, c^{\nu}\right) \\
c^{j}+\frac{c^{\nu}}{1+r\left(1-\tau_{k}\right)}=\mathrm{W}
\end{array}\right.
$$

HYPOTHÈSE 2. Les consommations aux deux dates sont des biens normaux : elles croissent avec la richesse actualisée du consommateur.

Considérons une situation stationnaire où la valeur du capital est $k$. Le taux d'intérêt et le salaire sont alors donnés par les formules (1), et on déduit de (4) le revenu intertemporel fonction du stock de capital $k$ et du taux de remplacement du régime de retraite $\theta$. En remplaçant les arguments $r$ et $\mathrm{W}$ en fonction de $k$ et $\theta$ dans les décisions optimales $C^{j}$ et $\mathbf{C}^{\nu}$, on obtient ainsi des demandes $\mathrm{D}^{j}(k, \theta)$ et $\mathrm{D}^{v}(k, \theta)$, que nous qualifierons de demandes de long terme. Ce sont les demandes qui sont pertinentes pour étudier les équilibres stationnaires.

Une trajectoire stationnaire est un équilibre si elle satisfait la contrainte emplois-ressources, lorsque les décisions des agents sont optimales étant donné les prix des facteurs associés à la valeur du capital. Comme on doit avoir $s=k(1+n)$, ceci conduit à la définition :

DÉFINITION. Le niveau de capital $k$ soutient un équilibre stationnaire associé

à des dépenses publiques $g$ si

$$
g+\mathrm{D}^{j}(k, \theta)+\frac{\mathrm{D}^{v}(k, \theta)}{1+n}+(n+\delta) k=f(k)
$$

Les niveaux de la dette que l'on peut dériver de la contrainte de budget des consommateurs et de l'État sont égaux. En effet, on peut vérifier qu'on a toujours la loi de Walras : en utilisant l'hypothèse de rendements constants $(f(k)=w+(r+\delta) k)$, la somme des contraintes de budget du jeune, du vieux (multipliée par $1 /(1+n)$ ) et de l'État est identique à la contrainte emploisressources.

1. Notons que l'on pourrait considérer d'autres politiques budgétaires, qui se traduiraient plus généralement par des dépenses publiques fonction de $k$ et $\theta$. 


\section{La règle d'argent}

Dans l'analyse traditionnelle sans distorsions fiscales (cas $\tau_{l}=\tau_{k}=0$ ), le stock de capital de la règle d'or, $k^{*}$, joue un rôle fondamental. Il est tel que le rendement net du capital est égal au taux de croissance de la population :

$$
n+\delta=f^{\prime}\left(k^{*}\right)
$$

Si le stock de capital est celui de la règle d'or, l'économie est en une situation optimale de long terme. De plus, toute intervention de l'État - et notamment toute modification du taux de cotisation retraite sans effet sur l'offre de travail - peut être neutralisée par les agents lorsqu'il n'y a pas de contraintes d'endettement. En présence de distorsions fiscales sur les revenus du capital, on conserve cette propriété de neutralité, mais en une autre valeur du stock de capital que celle de la règle d'or : c'est la valeur pour laquelle le rendement net du capital après impôt est égal au taux de croissance de la population. Nous l'appellerons, par analogie, le stock de capital de la règle d'argent, que nous noterons $\bar{k}$. Il est défini par :

$$
n=\left(1-\tau_{k}\right) r=\left(1-\tau_{k}\right)\left[f^{\prime}(\bar{k})-\delta\right]
$$

Dès lors que les revenus du capital supportent effectivement un impôt, la concavité de la fonction de production implique donc que le stock de capital de la règle d'argent est inférieur au stock de capital de la règle d'or.

À la règle d'argent la somme des revenus actualisés de l'agent est indépendante du taux $\theta$ de la répartition. L'interprétation est simple : la cotisation au régime de retraite s'assimile à un placement forcé de rendement égal au taux de croissance de la population. Dans le cas de la règle d'argent, ce rendement est identique à celui des actifs financiers. En l'absence de contrainte d'endettement, le système est neutre puisque tout accroissement de la cotisation peut être annulé par un emprunt d'un égal montant.

$\grave{A}$ quelles conditions le stock de la règle d'argent $\bar{k}$ est un équilibre ? La contrainte de budget de l'État est indépendante du montant de la dette, comme on le voit directement sur l'équation (6) : puisque le montant de la dette par tête est constant, l'accroissement de dette associé au mouvement démographique permet de payer exactement les charges d'intérêt. Le déficit primaire de l'Etat est nul et le niveau des dépenses publiques $\bar{g}$ est fixé par les recettes fiscales, indépendamment du taux de cotisation retraite ${ }^{1}$.

Ces propriétés de neutralité ont une portée plus générale, dès lors que la puissance publique peut répliquer par des impôts ou des subventions les opé-

1. On retrouve ce résultat par le calcul. Si $n=r\left(1-\tau_{k}\right)$ la demande agrégée est exactement égale au revenu intertemporel, soit ici : $\bar{w}\left(1-\tau_{l}\right)$. L'équation d'équilibre devient,

$$
\bar{g}+\vec{w}\left(1-\tau_{l}\right)+(\delta+n) \bar{k}=f(\bar{k})
$$

soit, en utilisant l'égalité $f(k)=w+(r+\delta) k$ :

$$
\bar{g}=\bar{w} \tau_{l}+\bar{r} \tau_{k} \bar{k}
$$


rations associées à la retraite par répartition, comme le montrent Belan et Pestieau [1997] et Pestieau et Possen [1997]. On peut alors substituer à la retraite par répartition un impôt sur les jeunes, une subvention aux retraités en augmentant le niveau de la dette publique de la somme correspondante.

Outre ses propriétés de neutralité, le stock de la règle d'argent joue un rôle important dans l'étude des équilibres. En effet, savoir si une variation du taux de cotisation augmente ou non le revenu intertemporel, à capital donné, c'est-à-dire à facteurs fixés, dépend uniquement de la position du stock de capital par rapport à celui de la règle d'argent :

PROPOSITION 0. Le revenu intertemporel est indépendant de $\theta$ lorsque le stock de capital est celui de la règle d'argent, $k=\bar{k}$. Il est décroissant avec $\theta$ pour un stock de capital inférieur $(k<\bar{k})$ et croissant pour un stock supérieur $(k>\bar{k})$.

La proposition 0 est très simple à démontrer. Si le stock de capital est plus faible que celui de la règle d'argent, $k<\bar{k}$, alors $r\left(1-\tau_{k}\right)>n$ : d'après (4), une augmentation de $\theta$ est équivalente à une baisse de revenu actualisé, associée à la différence de rendement entre placement financier et taux de croissance de la population. C'est l'idée familière dans le débat actuel que la retraite par capitalisation est préférable à la répartition. Cependant, ce raisonnement suppose un stock de capital constant. Notre analyse, qui est une analyse de long terme, prend en compte la modification des comportements et de l'équilibre associée à une variation du taux de cotisation.

\section{Existence et multiplicité}

Avant de procéder à la statique comparative des équilibres, il nous faut examiner leur existence et leur éventuelle multiplicité. Il est utile d'introduire la fonction de demande agrégée $D$ et celle d'excès de demande $Z$, définies de $\mathbb{R}^{+} \times \mathbb{R}^{+}$dans $\mathbb{R}$ respectivement par :

$$
\mathrm{D}(k, \theta)=\mathrm{D}^{j}(k, \theta)+\frac{\mathrm{D}^{\nu}(k, \theta)}{1+n}
$$

et

$$
\mathrm{Z}(k, \theta)=\mathrm{D}(k, \theta)+(n+\delta) k-f(k)
$$

Par construction, $k$ est un stock de capital associé à un équilibre stationnaire avec dépenses publiques $g$ si et seulement si :

$$
Z(k, \theta)+g=0
$$

L'existence et les propriétés des équilibres reposent sur la forme de la fonction $\mathrm{Z}$. Sous les hypothèses $\mathrm{H} 1$ et $\mathrm{H} 2$, le comportement au bord du domaine de $Z(\cdot, \theta)$ est simple : $Z$ est non négatif pour $k$ proche de 0 et lorsque $k$ tend vers l'infini. Puisque les dépenses publiques $g$ sont positives, $Z+g$ est positif, comme $\mathrm{Z}$, aux bornes du domaine. Le nombre d'équilibres dépend alors de la forme de $Z$, et génériquement il y a un nombre pair (éventuellement nul) de 
racines à l'équation d'équilibre. Lorsque les dépenses publiques ne sont pas trop élevées, il existe donc génériquement au moins deux équilibres ${ }^{1}$.

\section{L'ANALYSE DES ÉQUILIBRES STATIONNAIRES}

L'intérêt d'une modification du régime de retraite dans le long terme dépend in fine du bien-être atteint. Pour l'évaluer, nous étudions d'abord comment varient les stocks de-capital d'équilibre, quand, à dépenses publiques constantes, le niveau du taux de remplacement $\theta$ est modifié. Ensuite, nous déterminons dans quels cas il est bénéfique d'augmenter ou de diminuer le stock de capital.

\section{L'impact du taux de cotisation sur les équilibres}

Nous considérons l'impact d'une modification à la marge de $\theta$ sur l'équilibre en suivant l'équilibre par continuité. La dérivée par rapport à $\theta$ d'une des solutions de l'équation d'équilibre :

$$
\mathrm{Z}(k, \theta)+g=\mathrm{D}(k, \theta)+(n+\delta) k-f(k)+g=0
$$

s'évalue alors par le théorème des fonctions implicites : $Z_{\theta} d \theta+Z_{k} d k=0$, où l'indice fait référence à la variable de différenciation.

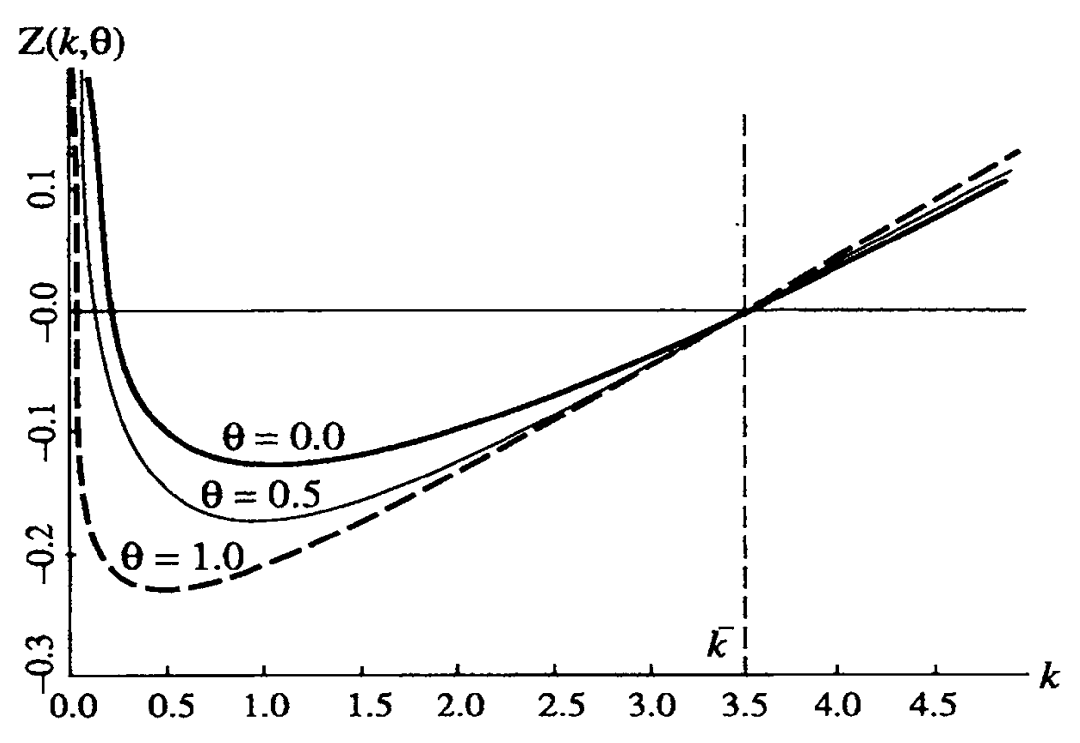

Le sens de variation de $k$ dépend du signe des dérivées de $Z$ par rapport à $\theta$ et par rapport à $k$. Le signe de $Z_{\theta}$ est aisément déterminé par la proposition 0 :

1. Une condition suffisante pour qu'il n'y ait jamais plus de deux équilibres est que l'excès de demande $Z(k, \theta)$ soit d'abord décroissante puis croissante en $k$. Sous $\mathrm{H} 1$, la production nette par tête $f(k)-(n+\delta) k$ est concave. Il suffirait que la demande D soit une fonction convexe en le stock de capital, mais nous n'avons pas trouvé de système d'hypothèses simple sur la fonction d'utilité qui garantisse cette propriété. 
seule la demande $D$ dépend de $\theta$, et ce à travers le revenu intertemporel. Sous $\mathrm{H} 1$, la demande est croissante avec le revenu. D'après la proposition $0, \mathrm{Z}$ est décroissante $^{1}$ en $\theta$ si et seulement si $k<\bar{k}$. Pour ce qui concerne $Z_{k}$ une analyse supplémentaire s'impose. Pour l'instant énonçons les propriétés de statique comparative des équilibres en fonction du signe de $\mathrm{Z}_{k}$.

PROPOSITION 1 : Soit $k(\theta)$ une solution à l'équation $\mathrm{Z}(k, \theta)=g$. Réduire à la marge le taux de cotisation $\theta$

1. accroît le stock de capital à l'équilibre si $k(\theta)<\bar{k}$ et $\mathrm{Z}_{k}<0$ ou bien si $k(\theta)>\bar{k}$ et $\mathrm{Z}_{k}>0$;

2. réduit le stock de capital si $k(\theta)<\bar{k}$ et $\mathrm{Z}_{k}>0$ ou bien si $k(\theta)>\bar{k}$ et $\mathrm{Z}_{k}<0$.

Démonstration. La proposition découle de ce que $Z$ est décroissante (croissante) par rapport à $\theta$ si $r\left(1-\tau_{k}\right)>n\left(r\left(1-\tau_{k}\right)<n\right)$, c'est-à-dire lorsque $k(\theta)<\bar{k}(k(\theta)>\bar{k})$.

Sur la figure 1 nous avons représenté avec un cas d'école ${ }^{2}$ les graphes de la fonction d'excès de demande $k \rightarrow \mathrm{Z}(k, \theta)$ pour différentes valeurs de $\theta$, à taux $\left(\tau_{l}, \tau_{k}\right)$ fixés. Ces graphes passent tous par le même point associés à la règle $\mathrm{d}$ 'argent $(\vec{k},-\vec{g})$. Lorsque $\theta$ augmente, les courbes se déplacent vers le bas dans l'intervalle $[0, \bar{k}]$, vers le haut lorsque le stock de capital est supérieur à $\bar{k}$. En la plus petite des racines $k_{\text {min }}$, la dérivée de $\mathrm{Z}$ par rapport à $k$ est négative, alors qu'elle est positive en la plus grande $k_{\max }$. En $k_{\min }$, qui est inférieur au capital de la règle d'argent, réduire à la marge le taux de cotisation $\theta$ accroît le stock de capital. L'effet est dans le même sens en la plus grande racine $k_{\max }$, qui est supérieure au stock de capital de la règle d'argent.

La proposition 1 peut surprendre. Alors que l'on réduit la part des retraites financées par la répartition au profit de la capitalisation, et que les salariés vont

1. On peut être plus précis quant à la dépendance du stock de capital d'équilibre par rapport au taux de remplacement $\theta$, sous des hypothèses de différentiabilité. En additionnant les contraintes budgétaires du jeune et du vieux, cette dernière multipliée par $1 /(1+n)$, on obtient :

$$
\frac{\partial Z}{\partial \theta}=\frac{r\left(1-\tau_{k}\right)-n}{1+n} \frac{\partial(s+b)}{\partial \theta}
$$

En un équilibre de sous-investissement, $\left(r\left(1-\tau_{k}\right)-n\right)$ est positif, ou à la rigueur nul. La dérivée de l'épargne $(b+s)$ par rapport à $\theta$ est elle aussi proportionnelle à $\left(r\left(1-\tau_{k}\right)-n\right)$, ce qui rend la dérivée de $Z$ par rapport à $\theta$ proportionnelle au carré de la différence entre le rendement du capital après impôt et le taux de croissance de la population : l'impact d'une réduction de la répartition sur le stock de capital est beaucoup plus fort en valeur absolue en un équilibre éloigné de la règle d'argent.

2. La population vit deux périodes et croît de $2 \%$ par période. La fonction d'utilité du consommateur représentatif est $\log c^{j}+\log c^{\nu}$. La fonction de production est CobbDouglas : $f(k)=k^{\alpha}$ avec $\alpha=0,3$. Le capital se déprécie au taux de $10 \%$ par période. II n'y a pas d'impôt sur le salaire et les revenus du capital sont taxés au taux de $20 \%$. La courbe $Z$ est tracée dans trois cas : pas de retraite par répartition $(\theta=0)$, la retraite est égale à la moitié $(\theta=0,5)$ ou à la totalité $(\theta=1)$ du salaire d'activité. 
accroître leur épargne, le stock de capital de l'économie peut décroître. Les débats sur le financement des retraites évoquent la possibilité d'un effet d'éviction, les placements des fonds de capitalisation se substituant pour partie à des investissements déjà existants, mais ne mentionnent pas, du moins à notre connaissance, le risque que l'éviction soit si forte qu'elle aille jusqu'à une baisse du stock de capital ! Notre analyse met l'accent sur le rôle crucial de la demande agrégée de consommation des actifs et des retraités : c'est elle qui conditionne l'accroissement plus ou moins fort du stock de capital d'équilibre, et éventuellement l'éviction, et nous y reviendrons en détail dans la dernière partie.

Du point de vue de la pertinence de la réforme, ce n'est pas le stock de capital qui importe, mais le niveau d'utilité des agents à l'équilibre, sur lequel nous portons maintenant notre attention.

\section{L'impact du taux de cotisation sur le bien-être}

La proposition suivante permet de qualifier de sous-investissement les niveaux de capital inférieurs à celui de la règle d'argent.

PROPOSITION 2 : Supposons la fonction d'utilité intertemporellement séparable. Soit $k<\bar{k}$ une valeur du stock de capital d'équilibre associée à une dépense publique $g$. Toute variation de $\theta$ qui, à dépenses publiques constantes, conduit à une augmentation de $k$ accrô̂t le niveau d'utilité à l'équilibre.

Preuve. À des fins d'application ultérieure, nous donnons une preuve de cette propriété pour le cas où les agents vivent pendant $a=1, \ldots$, A périodes. On note $c^{a}$ la consommation de $1^{\prime}$ agent d'âge $a, u^{a}$ son utilité marginale instantanée. Considérons un équilibre associé aux dépenses $g$ avec un niveau de capital $k$. Il est caractérisé par l'égalité emplois-ressources :

$$
g+\sum_{a=1}^{\mathrm{A}} \frac{c^{a}}{(1+n)^{a-1}}=f(k)-(n+\delta) k
$$

et les conditions du premier ordre du consommateur :

$$
u^{a a}=\left(1+r\left(1-\tau_{k}\right)\right) u^{a+1}, \quad \text { où } \quad r=f^{\prime}(k)-\delta
$$

Supposons qu'en faisant varier $\theta$, on modifie $k, g$ restant fixé. La variation d'utilité est donnée par :

$$
\Delta u=\sum_{a=1}^{\mathrm{A}} u^{a} \Delta c^{a}=u^{\prime 1} \sum_{a=1}^{\mathrm{A}} \frac{\Delta c^{a}}{\left(1+r\left(1-\tau_{k}\right)\right)^{a-1}}
$$

où les variations des consommations vérifient :

$$
\sum_{a=1}^{\mathrm{A}} \frac{\Delta c^{a}}{(1+n)^{a-1}}=\left\{f^{\prime}(k)-(\delta+n)\right\} \Delta k=(r-n) \Delta k
$$

Supposons $\Delta k>0$. Montrons que si $\left(1-\tau_{k}\right) r>n(k$ inférieur au niveau $\bar{k}$ de la règle d'argent) la consommation s'accroît en début de vie, jusqu'à un âge $\bar{a}>1$, avant éventuellement de décroître jusqu'au décès. En effet, d'après (9), si $\Delta c^{a}$ est négatif, comme le taux d'intérêt diminue, $\Delta c^{a+1}$ est aussi négatif : dès 
que la consommation baisse à un âge, elle baisse à tous les âges supérieurs. D'après la contrainte emplois-ressources, la somme des variations de consommation actualisées au taux $(1+n)$ est positive, ce qui implique que $\Delta c^{1}>0$.

Posons $\lambda=(1+n) /\left(1+r\left(1-\tau_{k}\right)\right)$. L'hypothèse $k<\bar{k}$ implique $\lambda<1$. La variation d'utilité s'écrit alors :

$$
\begin{aligned}
\frac{\Delta u}{u^{\prime \prime}} & =\sum_{a=1}^{\mathrm{A}} \frac{\Delta c^{a}}{(1+n)^{a-1}} \lambda^{a-1} \\
& =\lambda^{\bar{a}} \sum_{a=1}^{\mathrm{A}} \frac{\Delta c^{a}}{(1+n)^{a-1}} \lambda^{a-1-\bar{a}}
\end{aligned}
$$

Or pour tout $a$, on a $\Delta c^{a}\left(\lambda^{a-1-\bar{a}}-1\right) \geq 0$. En effet, si $a<\bar{a}+1$, les deux termes $\Delta c^{a}$ et $\left(\lambda^{a-1-\bar{a}}-1\right)$ sont positifs et inversement, si $a \geq \bar{a}+1$, ils sont tous deux négatifs. On obtient donc que :

$$
\frac{\Delta u}{u^{\prime \prime}} \geq \sum_{a=1}^{\mathrm{A}} \lambda^{\bar{a}}\left\{\frac{\Delta c^{a}}{(1+n)^{a-1}}\right\}
$$

Le terme entre accolades est égal à la variation de consommation agrégée qui, par la contrainte emploi-ressources (10), est égal à $(r-n) \Delta k>0$, qui est positif.

Une adaptation simple de la preuve montre qu'un niveau de capital supérieur à celui de la règle d'or correspond à un sur-investissement: réduire le stock de capital augmente l'utilité le long de l'équilibre stationnaire. Par contre, nous ne pouvons rien dire quant aux niveaux intermédiaires entre la règle d'argent et la règle d'or à cause de la distorsion due à la taxation des revenus du capital.

Nous sommes maintenant en mesure d'examiner à la lumière des propositions 1 et 2 le problème de politique économique à l'étude : dans quelles situations la réduction du taux de cotisation est-elle bénéfique?

Supposons que l'économie est en un équilibre où le capital est inférieur à celui de la règle d'argent $\bar{k}$. Réduire le taux de cotisation améliore le bien-être de long terme si $\mathrm{Z}_{k}>0$, le réduit si $\mathrm{Z}_{k}<0$.

\section{LE PASSAGE À LA CAPITALISATION EST-IL BÉNÉFIQUE DANS LE LONG TERME ?}

L'analyse théorique nous donne les éléments nécessaires pour étudier les effets à long terme d'un glissement de la répartition vers la capitalisation. Techniquement, il s'agit d'évaluer les dérivées partielles dans l'égalité :

$$
\mathrm{Z}_{\theta} d \theta+\mathrm{Z}_{k} d k=0
$$

Économiquement, il faut savoir comment l'excès de demande agrégée varie avec le taux de remplacement du régime de répartition et avec le stock de capital. Cette analyse sera conduite en supposant que les agents vivent plus de deux 
périodes, modèle qui généralise le cas retenu jusqu'ici pour la commodité de l'exposé. Tous les actifs gagnent le même salaire, quel que soit leur âge, et les retraites sont indexées sur les salaires. L'unité de temps est l'année, L est la durée de vie, $l$ la longueur de la vie active. En transposant les notations introduites en (7), on note $C^{a}(r, W), a=1, \ldots, L$, le programme de consommation solution de :

$$
\begin{aligned}
& \max _{a=1}^{L} \frac{1}{(1+\rho)^{a-1}} u\left(c^{a}\right) \\
& \sum_{a=1}^{L} \frac{c^{a}}{\left(1+r\left(1-\tau_{k}\right)\right)^{a-1}}=\sum_{a=1}^{e} \frac{w\left(1-\tau_{l}\right)\left(1-\tau_{r}\right)}{\left(1+r\left(1-\tau_{k}\right)\right)^{a-1}} \\
& \quad+\sum_{a=l+1}^{L} \frac{\theta w\left(1-\tau_{l}\right)}{\left(1+r\left(1-\tau_{k}\right)\right)^{a-1}}=W
\end{aligned}
$$

où $\tau_{r}$ est le taux de cotisation au régime de retraite par répartition qui assure l'équilibre financier du régime. La demande de consommation agrégée à la date de référence s'obtient alors comme somme des demandes des agents vivants, les diverses générations étant pondérées par leur taille relative :

$$
\mathrm{D}\left(r\left(1-\tau_{k}\right), \mathrm{W}\right)=\sum_{a=1}^{\mathrm{L}} \frac{1}{(1+n)^{a}} \mathrm{C}^{a}\left(r\left(1-\tau_{k}\right), \mathrm{W}\right)
$$

Il est d'abord important de situer la valeur du capital par rapport à celle de la règle d'argent, puisque, d'après la proposition 0 , qui s'étend aisément au cas de plusieurs périodes, elle détermine l'impact de la variation de $\theta$ sur le revenu intertemporel, i.e. $\partial \mathrm{W} / \partial \theta$. Nous étudierons ensuite les deux termes, $\mathrm{Z}_{\theta}, \mathrm{Z}_{k}$ qui sont donnés respectivement par :

$$
\mathrm{Z}_{\theta}=\partial \mathrm{D} / \partial \mathrm{W} \times \partial \mathrm{W} / \partial \theta \text { et } \mathrm{Z}_{k}=\mathrm{D}_{k}-(r-n)
$$

\section{Le passage à la capitalisation augmente le revenu des ménages, toutes choses égales par ailleurs}

Il y a abondance d'études qui concluent que la productivité marginale nette du capital est aujourd' hui bien supérieure au taux de croissance de la population, augmenté du taux de progrès technique (cf. Abel et al. [1989] pour les ÉtatsUnis). Le stock de capital est donc inférieur à la valeur de la règle d'or. Cela ne permet pas a priori de le situer par rapport à la règle d'argent, qui est associée à une valeur du capital par tête inférieure dès lors que les revenus du capital sont taxés. Mais, selon les évaluations que l'on retient habituellement, la productivité marginale du capital après impôt reste supérieure à la somme du taux de croissance de la population et du taux de progrès technique. Ceci nous conduit à focaliser l'attention sur le cas où le stock de capital est inférieur à celui de la règle d'argent.

Plus concrètement, selon l'étude de Feldstein et Samwick [1996], le rendement du capital avant impôt $r$ serait d'environ $9 \%$ par an aux États-Unis sur le dernier siècle boursier. Il est, en espérance mathématique, très supérieur au 
rendement des cotisations du régime de répartition ${ }^{1}, 2,5 \%$, lui-même de l'ordre de grandeur du taux de croissance de l'économie (qui, avec nos notations, correspond à la somme du taux de croissance de la population $n$, et du taux de progrès technique).

On obtient des ordres de grandeur analogues à partir d'un calcul de dos d'enveloppe pour la France. Supposons que la fonction de production macroéconomique soit de type Cobb-Douglas à rendements constants, avec une élasticité de la production au capital productif de 0,3 . Choisissons le facteur multiplicatif pour qu'avec un capital productif de 15000 milliards, la valeur ajoutée soit de 8000 milliards, chiffres approximatifs de 1997. La productivité marginale (brute) du capital est alors déterminée : elle vaut $16 \%$. Il y a une grande incertitude sur le taux de dépréciation (ou son inverse, la durée de vie des équipements), qui est probablement entre 6 et $8 \%$. Le taux d'intérêt avant impôt serait donc compris entre 8 et $10 \%$. On prévoit par ailleurs un taux de croissance de la population de $0,5 \%$ par an pour les prochaines décennies, et un taux de progrès technique entre 1 et $1,5 \%$. On retrouve donc, avec une approche beaucoup plus indirecte, des ordres de grandeur voisins de ceux de Feldstein et Samwick.

Ainsi, toutes choses égales par ailleurs, c'est-à-dire sans prendre en compte les réactions des agents, le passage à la capitalisation engendre dans le long terme un accroissement de la richesse actualisée des participants à l'économie. Cet accroissement vient du différentiel $r\left(1-\tau_{k}\right)-n$, par le jeu des intérêts composés sur longue période : il est substantiel. Cet effet correspond au gain tant vanté du passage à la capitalisation. Chez Feldstein et Samwick [1997], ce gain est renforcé par le fait que la souscription au régime de retraite par capitalisation est obligatoire, et que les fonds de pension sont exemptés d'impôts sur les revenus du capital. Cependant, cet accroissement de richesse doit engendrer des variations de la demande et de l'épargne. À court terme, tant que le taux d'intérêt et le stock de capital restent proches de leur valeur initiale, il est vraisemblable que cet accroissement de richesse se traduise par un accroissement d'épargne. Mais ces mouvements initiaux ne préjugent pas de ce qui se passera à l'équilibre de long terme. La demande de biens supplémentaire va susciter une production accrue, et donc une accumulation de capital, qui entraînera une baisse du taux d'intérêt. La hausse de la production et des revenus, la baisse du taux d'intérêt auront des répercussions en retour sur la consommation. Ce sont ces mécanismes que nous examinons maintenant, à travers la dépendance de l'excès de demande par rapport à $\theta$ et $k$.

\section{La dépendance de l'excès de demande par rapport au taux de remplacement et au stock de capital}

La dépendance de l'excès de demande par rapport au taux de remplacement est donnée par la dérivée partielle $\mathrm{Z}_{\theta}$ :

$$
\mathrm{Z}_{\boldsymbol{\theta}}=\partial \mathrm{D} / \partial \mathrm{W} \times \partial \mathrm{W} / \partial \theta
$$

1. Les organismes de retraite retiennent un chiffre un peu plus bas, plutôt $2 \%$, aux États-Unis. 
Le deuxième terme du produit vient d'être étudié. Le premier serait égal à 1 si le taux de croissance de la population était égal au taux d'intérêt après impôt, d'après l'identité budgétaire. Sous l'hypothèse $\mathrm{H} 2$ que la consommation est un bien normal à toutes les dates, et lorsque le taux d'intérêt après impôt est supérieur au taux de croissance, il est supérieur à 1 :

$$
\begin{aligned}
\sum_{a=1}^{L} \frac{1}{(1+n)^{a}} \mathrm{C}_{\mathrm{w}}^{a}\left(r\left(1-\tau_{k}\right), \mathrm{W}\right) & > \\
& \sum_{a=1}^{\mathrm{L}} \frac{1}{\left(1+r\left(1-\tau_{k}\right)\right)^{a}} \mathrm{C}_{\mathrm{w}}^{a}\left(r\left(1-\tau_{k}\right), \mathrm{W}\right)=1
\end{aligned}
$$

Quant à la dépendance de l'excès de demande par rapport au stock de capital elle est donnée par: $\mathrm{Z}_{k}=\mathrm{D}_{k}-(r-n)$. $\mathrm{D}_{k}$ s'obtient à partir de la demande de consommation agrégée $\mathrm{D}\left(r\left(1-\tau_{k}\right), \mathrm{W}\right)$, après remplacement de $\mathrm{W}$ et $r\left(1-\tau_{k}\right)$ en fonction de $k$ dans (1). On a :

$$
\mathrm{D}_{k}=\mathrm{D}_{r} \frac{\partial r\left(1-\tau_{k}\right)}{\partial k}+\mathrm{D}_{\mathrm{w}} \frac{\partial \mathrm{W}}{\partial k}
$$

Nous avons déjà vu que $\mathrm{D}_{\mathrm{w}}$ est supérieur à 1 . Le facteur $\partial \mathrm{W} / \partial k$ combine de manière complexe les variations de salaires et de taux d'intérêt : une hausse de $k$ accroît les salaires, diminue le taux d'intérêt et donc augmente la richesse actualisée W. Au total, le deuxième terme est positif. La dérivée de la consommation par rapport au taux d'intérêt $D_{r}$ se décompose classiquement en un effet de substitution, négatif, et un effet revenu, négatif pour les ménages endettés, positif pour les autres. Elle est multipliée par un terme négatif. Tout compte fait, on ne peut donc rien dire d'assuré sur le signe de $Z_{k}$.

Il s'agit de formuler des conjectures, et c'est un exercice particulièrement délicat, sur le signe et l'ordre de grandeur de $Z_{k}$. À titre purement illustratif, nous avons calculé ces dérivées sur un exemple d'école, avec quelques caractéristiques proches de la situation française. La fonction de production est la fonction de Cobb Douglas évoquée plus haut. Le taux de dépréciation est de $8 \%$, de sorte que le taux d'intérêt avant impôt est de $16-8=8 \%$. La durée de vie active est de quarante ans, celle de la retraite de quinze ans. Le taux de croissance de la population est de $0,5 \%$ par an, le taux de progrès technique de $1 \%$. Le taux de remplacement du régime de retraite est de $55 \%$, et l'on détermine le taux de cotisation pour équilibrer le régime. Les taux d'imposition sur le travail et le capital sont respectivement de $30 \%$ et $20 \%$. Enfin les fonctions d'utilité sont logarithmiques, avec des taux de préférence pour le présent $\rho$ respectivement de $5 \%, 10 \%$ et $15 \%$. Les valeurs des dérivées, calculées au stock de capital actuel $^{1}$, sont présentées dans le tableau ci-contre.

Une baisse du taux de remplacement de $1 \%$, et corrélativement celle du taux de cotisation au régime par répartition, accrôit ex ante la richesse intertemporelle actualisée de la génération type de $-W_{\theta} / 100$, soit 4,2 milliards de francs, sur un total d'environ 1370 milliards. Le taux d'intérêt après impôt est ici de

1. Le niveau de la consommation agrégée n'est pas proche de l'observé dans cet exercice! 


\begin{tabular}{|l|c|c|c|}
\hline \multicolumn{1}{|c|}{$\rho$} & $5 \%$ & $10 \%$ & $15 \%$ \\
\hline $\mathrm{W}_{\theta}$ & \multicolumn{3}{|c|}{-420} \\
$\mathrm{D}_{\theta}$ & -1145 & -765 & -619 \\
$\mathrm{D}_{\mathrm{W}}$ & 2,7 & 1,8 & 1,5 \\
$\mathrm{D}_{\mathrm{w}} \frac{\partial \mathrm{W}}{\partial k}$ & 0,40 & 0,27 & 0,22 \\
$\mathrm{D}_{k}$ & $-0,19$ & 0,03 & 0,10 \\
$\mathrm{Z}_{k}$ & $-0,25$ & $-0,03$ & 0,04 \\
\hline
\end{tabular}

$0,80 \times 8=6,4 \%$, supérieur au taux de croissance de $1,5 \%$, et c'est la différence entre les deux, $4,9 \%$, par le jeu des intérêts composés, qui crée ce fort accroissement de richesse initial. Il serait encore plus élevé, si comme Feldstein et Samwick, on supposait que les sommes ainsi dégagées (et peut-être bloquées) étaient exemptées d'impôt sur le capital. Toujours ex ante, cette baisse du taux de remplacement se traduit par une hausse de la demande de consommation agrégée $-D_{\theta} / 100$, d'autant plus forte que la propension à consommer la richesse intertemporelle $D_{w}$ est élevée : cette propension ici croît avec la préférence pour le futur et avec l'horizon des agents. Elle est de 1,5 dans le cas d'une forte myopie $(\rho=15 \%)$, et de 2,7 pour $\rho=5 \%$.

Comment se transforme cette hausse initiale au nouvel équilibre de long terme ? L'application de la formule :

$$
\frac{d k}{d \theta}=-\frac{\mathrm{D}_{\theta}}{\mathrm{Z}_{k}}
$$

montre la très grande incertitude qui entoure la longue période. L'effet « taux d'intérêt » sur la consommation agrégée, $D_{r} \frac{\partial r\left(1-\tau_{k}\right)}{\partial k}$ (qui se lit comme la différence $D_{k}-D_{W} \frac{\partial W}{\partial k}$ dans le tableau), est très sensible au choix de la préférence pour le présent dans le modèle pur de cycle de vie ${ }^{1}$. De plus, il serait vraisemblablement profondément affecté par la prise en compte de contraintes d'endettement.

Il est intéressant de voir comment cette incertitude est traitée dans les études disponibles sur le sujet. Feldstein et Samwick [1997] dans une section intitulée « Endogenous Response of Personal Saving Behavior», prennent le parti de s'en tenir à l'impact initial du passage au régime de capitalisation. Leur régime de capitalisation est obligatoire, et ils jugent qu'une réduction de l'épargne volontaire ne peut être que fort limitée. étant donné le niveau très bas des patrimoines financiers des ménages aux États-Unis. Ils négligent également les effets sur la consommation de la hausse des revenus actualisés. Leur étude est essentiellement une analyse d'équilibre partiel, retraçant les effets mécaniques

1. La dérivée $Z_{k}$ change de signe pour un taux de préférence pour le présent compris entre 10 et $15 \%$, ce qui correspond à un multiplicateur infini. Il y a très peu de chances de rencontrer une telle situation : elle correspond à un équilibre très « instable », où les deux racines $k_{\text {inin }}$ et $k_{\max }$ sont confondues. 
des mesures envisagées sur la séquence des revenus reçus par les différentes générations (c'est-à-dire correspond à l'analyse menée aux pages 825-826). Le seul effet en retour qui est chiffré est la baisse de taux d'intérêt qu'induirait une augmentation du stock de capital égale à l'actif du fonds de capitalisation, et son impact sur le niveau des pensions.

\section{CONCLUSION}

Contrairement à une présomption qui semble largement partagée par les spécialistes, notre analyse indique qu'il ne va pas de soi que, dans le long terme, un transfert à la marge de la répartition vers la capitalisation soit bénéfique. Dans nos économies, un telle opération accroît le bien-être dès lors qu'elle augmente le stock de capital d'équilibre, tant que le rendement du capital reste supérieur à la somme du taux de croissance de la population et du taux de progrès technique. Si l'on raisonne à stock de capital et taux d'intérêt fixés, l'effet de la mesure dans le long terme, quand on n'a plus à se soucier de financer la transition, est bien d'augmenter le revenu intertemporel de la génération type : la consommation, somme des demandes des agents de tous âges, s'accroît de ce fait. Mais il y a des effets en retour : les variations induites du stock de capital et du taux d'intérêt sont difficiles à anticiper et peuvent inverser le sens de la variation initiale. Le paramètre crucial est l'élasticité de la demande de consommation agrégée par rapport au taux d'intérêt. Dans le modèle de cycle de vie, le profil de consommation par âge est très sensible au taux d'intérêt et l'on ne dispose pas, à notre connaissance aujourd'hui, d'éléments théoriques ou empiriques qui permettent de trancher.

Notre étude laisse de côté deux éléments importants du débat. D'une part, nous nous sommes concentrés sur les situations de long terme, sans nous préoccuper de la transition. D'autre part, nous avons supposé l'offre de travail inélastique, de sorte que l'impôt sur les salaires et la cotisation au régime de répartition ne créent pas de distorsions.

Le sacrifice des générations qui supportent à la fois le poids de la répartition et celui de l'épargne nécessaire à leur propre retraite est inéluctable si l'équilibre initial est dynamiquement efficace. En l'absence d'imposition sur le capital, considérons un équilibre stationnaire avec un stock de capital inférieur à celui de la règle d'or (qui coïncide alors avec la règle d'argent). Depuis les travaux de Gale [1973], il est bien connu que cet équilibre est dynamiquement efficace : étant donné le stock de capital de la date 0 , il n'est pas possible de trouver un sentier de l'économie qui avantage tout le monde, à niveau fixé de la dépense publique $^{1}$. Le passage d'un équilibre stationnaire avec régime de retraite par répartition à un autre équilibre stationnaire meilleur, avec un stock de capital productif supérieur, suppose donc de sacrifier le bien-être de générations au cours de la transition.

1. La démonstration de cette propriété dans un cadre d'une durée de vie quelconque finie utilise le système de prix intertemporel qui soutient l'équilibre : voir, par exemple, Mas-Colell, Whinston et Green [1995, p. 773]. 
S'il existe un impôt sur le capital et/ou si l'offre de travail est élastique de sorte que les prélèvements fiscaux et sociaux induisent des distorsions sur l'allocation d'équilibre, le raisonnement précédent ne tient plus. Il est alors théoriquement possible de passer d'un état stationnaire avec régime de répartition à un autre avec capitalisation sans réduire l'utilité de quiconque en cours de route par rapport au statu quo, ceci d'autant plus aisément que la cotisation retraite du régime de répartition crée une inefficacité en réduisant l'offre de travail. Ce sont des arguments utilisés dans certains travaux comme ceux de Kotlikoff, Smetters et Walliser. Il serait intéressant de prolonger notre étude afin de tester si la fragilité des conclusions basées sur des simulations, mise en évidence dans notre modèle simplifié, s'étend à ces situations.

\section{RÉFÉRENCES BIBLIOGRAPHIQUES}

Abel A., Mankrw G., Summers L. et Zeckauser R. [1989], « Assessing Dynamic Efficiency : Theory and Evidence », The Review of Economic Studies, 56, p. 1-20.

BAllEN J. et GIL J. [1996], « Transitional Effects of a Pension System Change in Spain », Mimeo.

Belan P. et Pestreau P. [1997], «Privatizing Social Security : a Critical Assessment », Core Discussion Paper, novembre.

DIAMOND P. [1965], « National Debt in a Neoclassical Growth Model », American Economic Review, 55, p. 1126-1150.

Feldstein M. et Samwick A. [1996], « The Transition Path in Privatizing Social Security », NBER Working Paper $n^{\circ} 5761$.

FeldsteIN M. et SAMWICK A. [1997], « The Economics of Prefunding Social Security and Medicare Benefits ", NBER Working Paper n 6055.

GALE D. [1973], « Pure Exchange Equilibrium of Dynamic Economic Models », Journal of Economic Theory, 5, p. 12-36.

HUANG H., IMrohoroglu S. et SARGENT T. [1996], « Two Computations to Fund Social Security », Mimeo.

Kotlukoff L., SMeTters K. et WALliser J. [1996], « Privatizing U.S. Social Security. A simulation Study », Mimeo.

Mas-Colell A., Whinston M.D. et GReen J.R. [1995], Microeconomic Theory, Oxford, Oxford University Press.

PestJeau P. et PosSen U.M. [1997], « Investing Social Security in the Equity Market : Does it Make a Difference », Mimeo. 